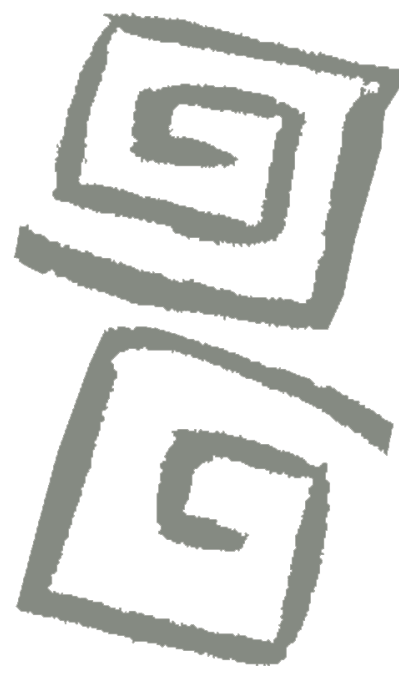

\title{
Análisis de la intervención de la política de comedores escolares y el rol en la nutrición saludable de niños y niñas de Córdoba, Argentina
}

\author{
An analysis of policy interventions regarding school \\ lunch programs and their role in the healthy nutrition of \\ children in Córdoba, Argentina
}

Daniela Moyano', Elizabeth Raquel Rodríguez ${ }^{2}$, Nilda Raquel Perovic ${ }^{3}$

${ }^{1}$ Autora de correspondencia. Magíster en Salud Pública. Investigadora, docente $y$ analista de políticas públicas, Escuela de Nutrición, Facultad de Ciencias Médicas, Universidad Nacional de Córdoba, Córdoba, Argentina. Departamento de Ciencias de la Salud, Universidad Nacional de la Matanza, Buenos Aires, Argentina. $\triangle$ iD

${ }^{2}$ Licenciada en Nutrición. Investigadora, Escuela de Nutrición, Facultad de Ciencias Médicas, Universidad Nacional de Córdoba, Córdoba, Argentina. $\triangle$ (iD)

${ }^{3}$ Doctora en Ciencias de la Salud. Investigadora y docente, Escuela de Nutrición, Facultad de Ciencias Médicas, Universidad Nacional de Córdoba, Córdoba Argentina. $\square$ (iD)
RESUMEN El objetivo fue analizar la intervención de la política de comedores escolares y el rol en la nutrición saludable de niños y niñas de Córdoba, Argentina en los años 2013 y 2018. Estudio descriptivo y trasversal. La muestra incluyó diez escuelas en 2013 y diez diferentes en 2018. Se realizó un relevamiento de comedores escolares y recordatorios alimentarios 24 hs a 341 niñas y niños. Los aportes nutricionales de los comedores escolares descendieron entre ambos periodos, en especial, en las escuelas insertas en contexto de mayor vulnerabilidad. También se observó una reducción significativa de la ingesta media de calcio y energía total. En las niñas y los niños asistentes a los comedores escolares se encontraron indicadores de desnutrición crónica, exceso de peso e ingestas de calcio, vitamina A y C deficitarios. En la asociación entre la asistencia al comedor escolar y el indicador baja talla/riesgo de baja talla, el odds ratio no fue estadísticamente significativa. Resulta necesario impulsar una política que busque de manera urgente mejoras de los indicadores de nutrición infantil, considerando un enfoque de derechos.

PALABRAS CLAVES Alimentación Escolar; Nutrición del Niño; Servicios de Alimentación; Argentina.

\begin{abstract}
The aim of this article is to analyze school lunch programs and their role in the healthy nutrition of children in Córdoba, Argentina from 2013 to 2018. A descriptive, cross-sectional study was conducted that included a sample of 10 schools in 2013 and 10 different schools in 2018. A survey of school lunch programs and 24-hour nutrition reminders was carried out with 341 children. The nutritional value of school lunch programs decreased over the study period, particularly among schools in contexts of greater social vulnerability. Significant reductions in average calcium and energy intake were also observed. Indicators of chronic malnutrition, overweight, and excess calcium and vitamin $\mathrm{A}$ and $\mathrm{C}$ intakes worsened among children attending school lunch programs. Regarding the association between school lunch program attendance and low height for age/risk of low height for age, the odds ratio was not statistically significant. We argue for the necessity of promoting policies that seek urgent improvements in child nutritional indicators from a rights-based perspective.
\end{abstract}

KEY WORDS School Lunches; Child Nutrition; Food Services; Argentina. 


\section{INTRODUCCIÓN}

En América Latina y el Caribe, el exceso de peso infantil se presenta como un panorama alarmante. El sobrepeso continúa aumentando y afecta al $7,3 \%$ de la población menor de 5 años, una cifra que supera el promedio mundial de 5,6\%(1). En Argentina, según datos recientes de la Segunda Encuesta Nacional de Nutrición y Salud (ENNyS 2), el 41,1\% de la población de 5 a 17 años presentó exceso de peso ${ }^{(2)}$.

Por otro lado, la baja talla sigue presente, teniendo estrecha relación con las desigualdades y la pobreza ${ }^{(1)}$. En Argentina, en el período 2018-2019 la baja talla afectó al 7,9\% de la población menor de 5 años $^{(2)}$. Como lo plantearon algunos autores, la malnutrición en sus múltiples formas se constituyen actualmente en un desafío para la salud pública ${ }^{(3)}$.

La Tercera Encuesta Mundial de Salud Escolar de 2018, que evalúa factores de riesgo en adolescentes de entre 13 y 15 años del país, mostró que el $19 \%$ presentaba sobrepeso, y un 2,6\% obesidad, cifra que se incrementó desde $2012^{(4)}$.

En Argentina, en las últimas décadas, se ha generado un importante proceso de reconocimiento de los derechos de niñas, niños y adolescentes, como la Convención Internacional de los Derechos del Niño de 1990, y la aprobación, en 2005, de la Ley 26061 de Protección Integral de los Derechos de $\mathrm{Ni}$ ños, Niñas y Adolescentes. Con relación a la alimentación como derecho, el país cuenta con el resorte jurídico de la Ley 25724, que dio origen al Programa de Nutrición y Alimentación Nacional y, más adelante, al Plan Nacional de Seguridad Alimentaria (PNSA), creado en 2003 con el objetivo de posibilitar el acceso de la población en situación de vulnerabilidad social a una alimentación adecuada, suficiente y acorde con las particularidades y costumbres de cada región del país. Dentro de su Artículo 13, se hace referencia a las expresas garantías de ejecución regular de los fondos destinados a comedores escolares por cada jurisdicción.
Además, se cuenta con la Ley 26396 que, en su Artículo 8, pone foco en la coordinación entre el Ministerio de Salud y el Ministerio de Desarrollo Social para garantizar que los comedores escolares velen por los aspectos nutricionales, poniendo especial énfasis en la corrección de las deficiencias o excesos de nutrientes, atendiendo las particularidades de la cultura alimentaria local.

Hoy, las políticas nutricionales impulsadas desde el Estado juegan un rol central en torno al alcance de la nutrición saludable en niños y niñas. Una de las políticas alimentarias más antiguas y con un amplio alcance en Argentina son los comedores escolares. Su implementación se encuentra a cargo de las provincias y/o municipios desde la descentralización de la década de 1990 y, según la evidencia, presenta importantes variaciones en los diferentes niveles de implementación ${ }^{(5)}$. Si bien estas políticas han sido protagonistas de las últimas décadas, las experiencias más antiguas se remontan a comienzos del siglo XX, como es el caso del Programa de Alimentación Escolar en Uruguay. Otros programas se crearon en las décadas de 1950 y 1960 , como Merienda Escolar de Brasil, Comedores Escolares de Argentina y el Programa de Alimentación Escolar en Chile ${ }^{(6)}$.

Al analizar la bibliografía de los últimos años sobre comedores escolares a nivel mundial, regional y nacional se ponen en evidencia las barreras al intentar implementar comedores escolares saludables alineados con la política estatal ${ }^{(7)}$. Se destacan las dificultades en cumplir con lo estipulado por la regulación ${ }^{(8,9)}$ y los factores que condicionan la definición del menú(10). Los estudios encontrados son consistentes en mostrar una calidad poco óptima de los servicios alimentarios brindados con déficit marcados en el aporte de micronutrientes y energía $a^{(9,11,12,13,14)}$. Algunos autores plantean que, en América Latina, los escenarios nutricionales de doble carga de malnutrición se ven plasmados desde los comedores escolares ${ }^{(15,16,17)}$.

Sin embargo, existen antecedentes que indican que intervenciones saludables en el ámbito de los comedores escolares podrían tener impactos positivos en la selección de 
alimentos ${ }^{(18)}$, serían efectivos para reducir el riego de obesidad ${ }^{(8,19)}$, influir favorablemente en la disminución del consumo de alimentos poco saludables y un aumento del consumo de frutas ${ }^{(19)}$ que pueden ser sostenibles en el tiempo ${ }^{(20)}$. Sin embargo, en el contexto local, durante los años 2013 y 2018 en los que se desarrolló este trabajo, no existieron aparentes iniciativas estatales en el marco de los comedores escolares más allá de la prestación alimentaria.

Existen algunos antecedentes tanto internacionales como nacionales que muestran que la alimentación de niñas y niños que asisten a comedores escolares no alcanza las metas nutricionales en la infancia ${ }^{(12,21,22)}$, y presenta un déficit en la ingesta de vegetales, más allá que la oferta teórica de los comedores escolares sea adecuada ${ }^{(23)}$ como también el caso de frutas y legumbres ${ }^{(24)}$.

En Argentina, la implementación de comedores escolares está a cargo de las provincias y/o municipios ${ }^{(25)}$, y algunos datos estarían indicando que aproximadamente 4,5 millones de niños y niñas, la mayoría de sectores vulnerables, se alimentan en la escuela $^{(25)}$. Las escasas experiencias evaluadas sobre comedores escolares a nivel regional $y$, especialmente, en América de Sur, hacen muy difícil sustentar el impacto real de esta política en la nutrición de la población destinataria. En el país, son pocos los antecedentes publicados, entre los que se encuentra una evaluación realizada en el año 1990(26), de la cual ya han trascurrido tres décadas. Sin investigaciones evaluativas, hay pocas posibilidades de una mejora continua en la gestión y pocas probabilidades de verificar el cumplimiento de los objetivos y metas ${ }^{(27)}$.

Este trabajo se encuentra enmarcado en la tesis de doctorado de la primera autora, titulada "La política pública alimentaria y nutricional destinada a la población infantil: un análisis de la implementación y del impacto de los Comedores Escolares de Córdoba, Argentina". El presente estudio tuvo como objetivo analizar la intervención de la política de comedores escolares y el rol en la nutrición saludable de niños y niñas de Córdoba, Argentina en los periodos 2013 y 2018.

\section{MÉTODOS}

\section{Diseño de estudio}

Se realizó un estudio descriptivo y transversal, a partir de fuente de datos primarios recolectados durante el año 2018, y de una base de datos secundaria con datos recolectados en $2013^{(28)}$. Se buscó realizar una comparación de variables de calidad nutricional de comedores escolares y de la ingesta de niños y niñas durante dos cortes temporales en un periodo de cinco años. Durante el año 2018 se profundizó en la comparación entre asistentes y no asistentes a comedores escolares con incorporación de indicadores de estado nutricional.

Contar con datos en dos momentos diferentes permitió realizar comparaciones en un periodo en el que se evidenciaron cambios importantes en la situación social y política del país, siendo un análisis útil e importante para el monitoreo y vigilancia en salud pública y de las intervenciones desde el Estado.

\section{Muestra y unidades de análisis}

El universo estuvo constituido por 37 escuelas municipales de Córdoba, Argentina, sus respectivos comedores escolares y los servicios brindados en dos días no consecutivos durante el mismo año de relevamiento. De este universo se seleccionó por conveniencia una muestra de 20 escuelas: diez fueron relevadas durante el año $2013^{(28)}$, y diez unidades diferentes en 2018. Para poder garantizar la factibilidad del estudio durante los dos periodos de relevamiento se implementó un muestreo no probabilístico, en el que se establecieron cuotas de escuelas según nivel socioeconómico, tomando como variable proxy el hacinamiento, según el Censo Nacional de Población, Hogares y Viviendas, $2010^{(29)}$. Considerando la factibilidad del estudio y la obtención de una muestra mínima que permita realizar los análisis propuestos, dentro de cada escuela, se seleccionó de manera aleatoria a niñas y niños, garantizando alcanzar cuotas mínimas compuestas por al 
menos 15 escolares de ambos sexos. Durante la recolección de $2013^{(28)}$ las y los escolares que se incluyeron solo fueron quienes asistían al comedor escolar mientras que, en 2018, considerando el contexto social, económico y político de país, se definió incorporar tanto asistentes como no asistentes al comedor escolar con el propósito de poder comparar ambos escenarios.

\section{Criterios de inclusión}

Se incluyeron las escuelas, los comedores escolares y los servicios brindados de localización urbana, que hayan brindado durante los días del relevamiento por lo menos dos de los servicios alimentarios frecuentes (desayuno, almuerzo y/o merienda). Se incluyeron niñas y niños de $4^{\circ}, 5^{\circ}$ o $6^{\circ}$ grado de las escuelas bajo estudio, con edades iguales o mayores a 8 años, y que asistieran al turno mañana o tarde.

Para determinar la inclusión de sujetos que asistieran al comedor escolar, se definió como criterio el autorreporte de asistencia el día antes del relevamiento, de por lo menos una de las comidas brindadas por el comedor. Dentro de los criterios de exclusión, se consideró a niñas y niños que presentaron alguna dificultad cognitiva, barreras idiomáticas o cualquier otra situación que no permitiera implementar el recordatorio alimentario 24 horas. Sin embargo, durante la etapa de campo no se detectaron situaciones que no permitieron avanzar con las entrevistas. Todas las niñas y todos los niños participantes contaron con el consentimiento informado firmado por el padre, la madre, o la persona responsable.

\section{Variables principales}

\section{Comedor escolar}

- Nutrientes críticos por déficit: promedio diario de vitamina $\mathrm{A}(\mu \mathrm{g})$, calcio $(\mathrm{mg})$, zinc $(\mathrm{mg})$, vitamina $\mathrm{C}(\mathrm{mg})$, fibra $(\mathrm{g})$, hierro $(\mathrm{mg})$, vitamina $\mathrm{D}(\mu \mathrm{g})$ y ácido fólico $(\mu \mathrm{g})$ aportados por los servicios de los comedores escolares.
- Nutrientes críticos por exceso: promedio diario de energía total (kcal), grasas saturadas (g), azúcares simples (g) y sodio (mg) aportados por los servicios de los comedores escolares.

\section{Ingesta de niños y niñas}

- Ingesta según los grupos de alimentos propuestos en las Guías Alimentarias para la Población Argentina (GAPA) de 2016 ${ }^{(30)}$. Categorías: Aceites, frutas secas y semiIlas; verduras y frutas; legumbres, cereales, papa, pan y pastas; leche, yogur y quesos; carnes y huevos; opcionales, dulces y grasas; $\operatorname{agua}^{(30)}$.

- Ingesta de nutrientes críticos por déficit por diferentes vías (alimentos y bebidas): consumo diario promedio de vitamina A $(\mu \mathrm{g})$, calcio $(\mathrm{mg})$, zinc $(\mathrm{mg})$, vitamina $\mathrm{C}(\mathrm{mg})$, fibra $(\mathrm{g})$, hierro $(\mathrm{mg})$, vitamina $\mathrm{D}(\mu \mathrm{g})$ y ácido fólico $(\mu \mathrm{g})$.

- Ingesta de nutrientes críticos por exceso por diferentes vías (alimentos y bebidas): consumo diario promedio de energía total (kcal), grasas saturadas (g), azúcares simples (g) y sodio (mg).

- Alcance (\%) de la media de ingesta total de energía y nutrientes críticos por déficit, de las metas nutricionales saludables: vitamina $\mathrm{A}(\mu \mathrm{g})$, calcio $(\mathrm{mg})$, zinc $(\mathrm{mg})$, vitamina $C(m g)$, fibra $(\mathrm{g})$, hierro $(\mathrm{mg})$, vitamina D $(\mu \mathrm{g})$ y ácido fólico $(\mu \mathrm{g})$. Categorías: alcanza/no alcanza.

- Alcance (\%) de la media de ingesta total de energía y nutrientes críticos por exceso, de las metas nutricionales saludables: energía total (kcal), grasas saturadas (g), azúcares simples (g) y sodio (mg). Categorías: alcanza/no alcanza.

- Cobertura de nutrientes por parte de la política de comedores escolares: cantidad (valores absolutos y porcentajes (\%) de cobertura) de nutrientes críticos por déficit y por exceso que aportan los servicios alimentarios de comedores escolares (alimentos y bebidas ofrecidas) que representan la ingesta total de nutrientes de niñas y niños. 
Estado nutricional: Categorías e indicadores

- Puntuación Z talla/edad, Z IMC/edad y Z peso/talla.

- Estado nutricional según Z-score IMC/edad (muy bajo peso; bajo peso; alerta de bajo peso, peso adecuado; alto peso y muy alto peso).

- Estado nutricional según Z-score peso/edad (desnutrición aguda severa; desnutrición aguda moderada; riesgo de desnutrición aguda; peso adecuado; riesgo de sobrepeso o sobrepeso; obesidad).

- Estado nutricional según Z-score talla/edad (muy baja talla; baja talla; alerta de baja taIla; talla adecuada y alta talla).

\section{Covariables}

- Observación (momento del relevamiento). Categorías: primer relevamiento (año 2013); segundo relevamiento (año 2018).

- Sexo (masculino/femenino).

- Edad (años cumplidos) y para algunos análisis se definió en meses cumplidos, grado $\left(4^{\circ}, 5^{\circ}\right.$ y $\left.6^{\circ}\right)$.

- Turno de asistencia escolar (mañana/tarde).

- Asistencia al comedor escolar (Sí/No).

- Hacinamiento ${ }^{(29)}$ (Número de hogares con más de tres personas por cuarto, correspondiente al barrio en el que se inserta la escuela). Se definieron las categorías de hacinamiento bajo/medio/alto de acuerdo a los valores máximos de cada tercil de la variable continua hacinamiento. Categorías: Hacinamiento bajo: hasta 34 hogares; hacinamiento medio: hasta 106 hogares; hacinamiento alto: hasta 639 hogares.

\section{Técnicas e instrumentos de recolección de datos: Calidad alimentaria y nutricional de los comedores escolares}

Se realizó un relevamiento de datos primarios y de datos secundarios ${ }^{(28)}$. En ambos relevamientos se utilizó la misma metodología para las variables recolectadas en ambos períodos. Este instrumento consistió en una guía de observación directa y registro de todos los alimentos y las bebidas en todos los servicios alimentarios ofrecidos en dos días no consecutivos. Se incluyó el pesaje de raciones seleccionadas al azar, utilizando una balanza electrónica portátil con capacidad de hasta $3 \mathrm{~kg}$; precisión $\pm 1 \mathrm{~g}$. A partir de los datos alimentarios obtenidos, se realizaron estimaciones de cantidad de nutrientes utilizando bases de datos de composición nutricional de Latinfoods, Argenfoods, el Sistema de Análisis y Registro de Alimentos (SARA), Etiquetado nutricional de industrias alimentarias de Argentina publicadas en páginas web y Departamento de Agricultura de los Estados Unidos (Versión 28).

Para la ingesta alimentaria y nutricional y el alcance de metas nutricionales saludables, se empleó el método de recordatorio alimentario de 24 horas $^{(31,32)}$, validado para niñas y niños mayores de 8 años. A fin de minimizar posibles sesgos, se empleó la técnica de cuatro pasos de Gibson ${ }^{(32)}$, el uso de modelos visuales de alimentos validados, recetas estandarizadas e instrumentos caseros (cucharas, tazas u otros). Se realizaron dos observaciones no consecutivas a una submuestra del $15 \%$ por escuela y se empleó el método "multiple source method" para ajustar estadísticamente la ingesta.

Se estimó la cantidad de energía y nutrientes críticos por déficit y exceso, y se utilizaron las bases de composición nutricional antes mencionadas. Se determinó el alcance de las metas nutricionales saludables tomando como referencia la ingesta dietética de referencia (dietary reference intakes) recomendada por el Instituto de Medicina de Estados Unidos ${ }^{(33)}$, OMS/FAO $2001^{(34,35)}$ y $2003^{(36)}$. Se definieron los rangos etarios de energía propuestas por la OMS/FAO $2001^{(34)}$ y, para su aplicación, se tomó como peso de referencia para niñas y niños el percentil 50 según edad y sexo, según la Guía para la evaluación del crecimiento, publicada por la Sociedad Argentina de Pediatría en $2013^{(37)}$. El factor de actividad considerado para el cálculo fue el correspondiente para la actividad física moderada. En el caso de los micronutrientes en los que no existían datos sobre recomendaciones, se utilizaron los valores de ingesta adecuada. 
Para definir el estado nutricional de niñas y niños se siguieron los lineamientos de OMS y de la Guía para la evaluación del crecimiento físico, de la Sociedad Argentina de Pediatría ${ }^{(37)}$, para implementar un instrumento de recolección de mediciones antropométricas. Previo a la implementación del relevamiento, el equipo de investigadores recibió una capacitación con la finalidad de estandarizar las mediciones. Para estimar el peso se utilizó una báscula de plataforma con precisión de 100 g, y para la talla se utilizó un estadímetro. Para el cálculo de indicadores del estado nutricional de niñas y niños se utilizó el Software Anthro de la OMS.

\section{Análisis de los datos}

Se realizó un análisis descriptivo de las variables principales y las covariables de interés, utilizando medidas de tendencia central y dispersión (media); desvío estándar, mediana y percentiles 25 y 75 para las variables continuas y frecuencias absolutas y relativas para los datos categóricos.

Se evaluó la distribución de los datos continuos (nutricionales) por medio de métodos gráficos (histograma) y la prueba de Shapiro-Wilk para contrastar la normalidad de la distribución de los datos y, para el análisis de las varianzas, se utilizó la prueba de Bartlett. En todos los indicadores antropométricos siempre se utilizaron datos estandarizados a través de la puntuación Z.

Para explorar posibles asociaciones entre variables dicotómicas de alcance de metas nutricionales saludables y la asistencia al comedor escolar se empleó un análisis multivariado a través de modelos de regresión logística (melogit), tomando como efecto variable las escuelas y como efectos fijos las variables provenientes de niñas y niños. Los resultados se expresan en odds ratio crudas y ajustadas por potenciales confusores. La multicolinealidad de las variables independientes se verificó mediante el factor de inflación de la varianza. Se exploraron posibles interacciones que no fueron estadísticamente significativas $(p>0,05)$. Para la bondad de ajuste se utilizó la curva ROC y test de Hosmer-Lemeshow como parámetros de discriminación y calibración del modelo. En todos los casos con intervalos de confianza del $95 \%$ y un valor de significancia estadística de $p<0,05$. Para todos los análisis se utilizó el software estadístico Stata versión 14.0.

\section{Aspectos éticos}

En el presente estudio se garantizaron todos los requerimientos éticos a nivel nacional. Se solicitó el consentimiento a madre/ padre/tutor de los niños y las niñas participantes según la normativa nacional vigente del Código Civil y Comercial de la Nación 2016 -respecto de la escala de edades para la firma del consentimiento informado- y los requerimientos de los comités de ética locales. El protocolo de la base secundaria (2013) fue evaluado por el Comité de Ética en Investigación del Hospital Nacional de Clínicas, Universidad Buenos Aires y, en 2018, por el Comité Institucional de Ética de las Investigación en Salud del Hospital Nacional de Clínicas, Universidad Nacional de Córdoba (RHNC N³575/2018).

\section{RESULTADOS}

La muestra de servicios alimentarios del Comedor escolar en el primer relevamiento fue de $n=58$ y en el segundo relevamiento fue $n=57$. La muestra total de niños y niñas durante los dos periodos de recolección de datos fue de $n=341$, que consistió en 150 niñas y niños en el primer relevamiento y 191 en el segundo relevamiento. Como se muestra en la Tabla 1, los indicadores sociodemográficos, en las dos cortes bajo análisis, siguieron una estructura similar, con un predominio del subgrupo de niñas, con edades iguales y/o mayores a 10 años, pertenecientes a cursos menores de $6^{\circ}$ grado y al turno mañana.

En la muestra global, y tomando como referencia los grupos de alimentos propuestos por las Guías alimentarias para la población argentina de 2016, se pudo constatar 
Tabla 1. Características sociodemográficas de la muestra de niñas y niños de escuelas municipales de Córdoba, Argentina. Años 2013 y 2018.

\begin{tabular}{|c|c|c|c|c|}
\hline \multirow[t]{2}{*}{ Características } & \multicolumn{2}{|c|}{$\begin{array}{l}\text { Primer relevamiento } 2013 \\
\qquad(\mathrm{n}=150)\end{array}$} & \multicolumn{2}{|c|}{$\begin{array}{l}\text { Segundo relevamiento } 2018 \\
\qquad(\mathrm{n}=191)\end{array}$} \\
\hline & $\mathrm{n}$ & $\%$ & $\mathrm{n}$ & $\%$ \\
\hline \multicolumn{5}{|l|}{ Sexo } \\
\hline Femenino & 88 & 58,6 & 118 & 61,8 \\
\hline Masculino & 62 & 41,3 & 73 & 38,2 \\
\hline \multicolumn{5}{|l|}{ Edad } \\
\hline Menor a 10 años & 59 & 34,7 & 101 & 44,5 \\
\hline Mayor 0 igual a 10 años & 111 & 65,3 & 126 & 55,5 \\
\hline \multicolumn{5}{|l|}{ Grado } \\
\hline Hasta $5^{\circ}$ grado & 92 & 54,1 & 127 & 66,5 \\
\hline Más de $5^{\circ}$ grado & 78 & 45,9 & 64 & 33,5 \\
\hline \multicolumn{5}{|l|}{ Turno de asistencia escolar } \\
\hline Mañana & 130 & 76,5 & 101 & 52,9 \\
\hline Tarde & 40 & 23,5 & 90 & 47,1 \\
\hline
\end{tabular}

que, del total de la muestra, los alimentos más consumidos pertenecieron al grupo de opcionales $(33 \%)$ y al grupo de legumbres, cereales, papa, pan y pastas ( $21 \%)$. Un $17 \%$ de los alimentos bajo análisis perteneció al grupo al de verduras y frutas, un $8 \%$ al de leche, yogur y quesos, un $8 \%$ carnes y huevos, $6 \%$ fue agua, un $7 \%$ perteneció al grupo de aceites, frutas secas y semillas.

Entre los dos puntos de corte, la mayoría de las medias de nutrientes críticos por déficit, en especial en el zinc, vitamina $C$, hierro y ácido fólico, presentaron una reducción del aporte de los servicios alimentarios de comedores escolares. Por ejemplo, en la media de hierro de la porción brindada por el comedores escolares pasó de 3,06 [IC95\% $(1,96-4,16)]$ en 2013 a 2,16 en 2018 [IC95\% (1,57-2,76)]; de ácido fólico de 142,09 [IC95\% (91,97192,21)] a 117,17 [IC95\% $(95,13-139,21)]$ y de vitamina C de 20,08 [IC95\% $(4,92-35,24)$ ] a 8,03 [IC95\% $(2,48-13,57)]$ dentro del estrato de hacinamiento alto (mayor vulnerabilidad). Esto se reflejó de manera similar en las medias de nutrientes considerados críticos por excesos, como los azúcares simples y energía. En el caso de la energía pasó de una media de 371,5 [IC95\% (264,02-479,05)] en 2013 a una media de 305,12 [IC95\% $(241,68-368,55)]$ dentro del estrato de mayor hacinamiento (Tabla 2).
Otro dato interesante es que en el subgrupo de comedores escolares insertos en contexto de nivel alto de hacinamiento, tanto en el 2013 como en el 2018, la mediana de calcio y de vitamina $D$ fue menor con respecto al subgrupo con nivel medio y/o bajo de hacinamiento (Tabla 2).

En la Tabla 3 se presenta un análisis de comparación entre las ingestas de los niños y niñas que formaron parte del primer relevamiento $(n=150)$ y el segundo relevamiento $(n=191)$ (Tabla 3). Se visualizó que en casi todas las medias de nutrientes críticos bajo análisis fueron más altas en el subgrupo que formó parte del relevamiento 2013 con respecto al 2018; sin embargo, las diferencias estadísticamente significativas se encontraron en la ingesta media total de calcio, donde la media en el 2013 fue de 573,49 [IC95\% (524,61-622,38)] y en el 2018 de 493,99 [IC95\% $(463,81-524,17)]$ y en la energía total con una media de 2480,59 [IC95\% (2333,38$2627,79)]$ y 2243,68 [IC95\% $(2146,99-$ $2340,38)]$, respectivamente. Solo en el caso de la fibra, se observó una ingesta levemente superior en el segundo relevamiento [media 9,83; IC95\% $(9,19-10,46)]$ versus el primer relevamiento [media 11,39; IC95\% (10,6212,15)] (Tabla 3). Un dato relevante es que tomando la mediana de nutrientes aportados 
Tabla 2. Mediana y percentiles 25 y 75 de nutrientes críticos de los alimentos y bebidas de los servicios alimentarios de comedores escolares según condición de hacinamiento y año de relevamiento. Escuelas municipales de Córdoba, 2013 y 2018.

\begin{tabular}{|c|c|c|c|c|c|c|c|c|c|c|c|c|}
\hline \multirow[t]{3}{*}{ Aporte nutricional } & \multicolumn{6}{|c|}{$\begin{array}{l}\text { Primer relevamiento } 2013 \\
\qquad(\mathrm{n}=58)\end{array}$} & \multicolumn{6}{|c|}{$\begin{array}{l}\text { Segundo relevamiento } 2018 \\
\qquad(\mathrm{n}=57)\end{array}$} \\
\hline & \multicolumn{2}{|c|}{ Hacinamiento bajo } & \multicolumn{2}{|c|}{ Hacinamiento medio } & \multicolumn{2}{|c|}{ Hacinamiento alto } & \multicolumn{2}{|c|}{ Hacinamiento bajo } & \multicolumn{2}{|c|}{ Hacinamiento medio } & \multicolumn{2}{|c|}{ Hacinamiento alto } \\
\hline & Mediana & p25-p75 & Mediana & p25-p75 & Mediana & p25-p75 & Mediana & p25-p75 & Mediana & p25-p75 & Mediana & p25-p75 \\
\hline \multicolumn{13}{|c|}{ Nutrientes críticos por déficit } \\
\hline Vitamina A $(\mu \mathrm{g})$ & 31,4 & $23,2-137,0$ & 49,5 & $22,4-164,5$ & 48,5 & $23,2-140,4$ & 41,7 & $19,3-101,1$ & 33,6 & $23,0-100,7$ & 42,0 & $24,9-67,5$ \\
\hline Calcio (mg) & 119,9 & $108,7-137,6$ & 134,0 & $100,5-189,5$ & 114,9 & $94,6-134,4$ & 134,4 & $92,6-191,0$ & 151,9 & $97,4-191,6$ & 126,7 & $80,5-214,2$ \\
\hline Zinc (mg) & 1,0 & $1,0-2,3$ & 1,6 & $0,9-3,7$ & 1,6 & $0,9-3,8$ & 1,1 & $0,8-1,3$ & 1,1 & $1,0-3,6$ & 1,2 & $0,9-2,8$ \\
\hline Vitamina C (mg) & 0,0 & $0,0-18,5$ & 0,0 & $0,0-37,5$ & 0,0 & $0,0-47,0$ & 0,0 & $0,0-5,5$ & 0,0 & $0,0-22,0$ & 0,0 & $0,0-6,4$ \\
\hline Fibra (g) & 1,2 & $1,1-3,4$ & 1,1 & $0,4-5,1$ & 1,1 & $0,4-4,3$ & 1,1 & $0,7-1,1$ & 1,3 & $1,1-2,4$ & 1,1 & $1,0-2,6$ \\
\hline Hierro (mg) & 1,4 & $1,4-3,4$ & 1,4 & $1,1-5,4$ & 3,3 & $1,4-5,1$ & 1,4 & $1,3-3,5$ & 1,4 & $1,4-4,3$ & 1,5 & $1,4-3,1$ \\
\hline Vitamina D $(\mu \mathrm{g})$ & 1,0 & $0,2-1,1$ & 0,9 & $0,2-1,1$ & 0,9 & $0,1-1,1$ & 0,23 & $0,1-1,3$ & 0,6 & $0,1-1,4$ & 1,2 & $0,2-2,0$ \\
\hline Ácido fólico $(\mu \mathrm{g})$ & 131,6 & $92,9-183,6$ & 131,3 & $70,0-184,0$ & 131,6 & $40,2-183,7$ & 132,6 & $21,60-135,99$ & 132,9 & $100,8-146,3$ & 133,4 & $83,5-153,2$ \\
\hline \multicolumn{13}{|c|}{ Nutrientes críticos por exceso } \\
\hline Azúcares simples (g) & 22,4 & $16,2-26,4$ & 27,0 & $20,0-32,2$ & 18,6 & $14,4-30,6$ & 15,9 & $13,61-20,49$ & 14,6 & $9,5-18,5$ & 17,4 & $9,4-22,9$ \\
\hline Sodio (mg) & 110,5 & $101,7-318,8$ & 160,8 & $95,6-397,2$ & 199,5 & $101,7-303,8$ & 139,3 & $93,73-212,01$ & 130,0 & $106,2-282,7$ & 150,4 & $105,1-221,8$ \\
\hline Grasas saturadas (g) & 2,6 & $1,6-6,0$ & 2,9 & $1,4-5,2$ & 2,4 & $1,4-5,7$ & 2,8 & $1,22-4,97$ & 2,3 & $1,6-6,4$ & 2,8 & $1,7-3,9$ \\
\hline Energía total (kcal) & 267,0 & $221,7-466,5$ & 322,9 & $214,9-518,4$ & 307,9 & $189,7-583,4$ & 239,3 & $181,77-334,79$ & 222,8 & $169,0-607,7$ & 261,0 & $169,2-385,8$ \\
\hline
\end{tabular}

Fuente: Elaboración propia.

IC 95\%= Intervalo de confianza del 95\%; p25-p75= percentiles 25 y 75; Hacinamiento ( $\mathrm{N}^{\circ}$ de hogares con más de tres personas por cuarto correspondiente al barrio donde se inserta la escuela). Esta variable se trabajó en el análisis como terciles de número de hogares con hacinamiento. Categorías: Bajo (tercil 1): hasta 34 hogares. Medio (tercil 2): más de 34 hasta 106 hogares. Alto (tercil 3): más de 106 hogares.

Tabla 3. Mediana y percentiles 25 y 75 de nutrientes críticos de la ingesta nutricional de niños y niñas y coberturas nutricionales por parte de comedores escolares y año de relevamiento. Escuelas municipales de Córdoba, 2013 y 2018

\begin{tabular}{|c|c|c|c|c|c|c|c|c|}
\hline \multirow[t]{2}{*}{$\begin{array}{l}\text { Ingesta nutricional de niños } \\
\text { y niñas }\end{array}$} & \multicolumn{4}{|c|}{$\begin{array}{l}\text { Primer relevamiento } 2013 \\
\qquad(\mathrm{n}=150)\end{array}$} & \multicolumn{4}{|c|}{$\begin{array}{l}\text { Segundo relevamiento } 2018 \\
\qquad(\mathrm{n}=191)\end{array}$} \\
\hline & Mediana & p25-p75 & $\begin{array}{c}\text { Cobertura (\%) } \\
\text { de la meta* }\end{array}$ & $\begin{array}{c}\text { Cobertura del } \\
\text { comedor escolar } \\
(\%)^{* *}\end{array}$ & Mediana & p25-p75 & $\begin{array}{c}\text { Cobertura (\%) } \\
\text { de la meta* }\end{array}$ & $\begin{array}{c}\text { Cobertura del } \\
\text { comedor escolar } \\
(\%)^{* *}\end{array}$ \\
\hline \multicolumn{9}{|l|}{ Nutrientes críticos por déficit } \\
\hline Vitamina $A(\mu \mathrm{g})$ & 375,8 & $228,9-559,5$ & 68,3 & 12,9 & 308,6 & $(199,5-464,4)$ & 56,1 & 13,5 \\
\hline Calcio (mg) & 523,2 & $319,7-735,1$ & 52,3 & 23,0 & 444,7 & $329,2-636,9$ & 44,5 & 30,3 \\
\hline Zinc (mg) & 11,0 & $8,3-13,9$ & 157,0 & 9,5 & 11,5 & $8,4-14,9$ & 164,7 & 10,2 \\
\hline Vitamina C (mg) & 96,7 & $36,4-493,4$ & 257,9 & 0,0 & 34,5 & $14,6-63,6$ & 91,9 & 0,0 \\
\hline Fibra (g) & 10,6 & $7,6-13,8$ & 37,2 & 10,6 & 9,2 & $6,6-12,1$ & 32,2 & 12,2 \\
\hline Hierro (mg) & 15,8 & $12,4-21,0$ & 126,7 & 13,4 & 16,1 & $12,0-22,1$ & 129,1 & 9,0 \\
\hline Vitamina D $(\mu \mathrm{g})$ & 2,0 & $0,6-3,8$ & 40,6 & 48,3 & 2,0 & $1,0-3,1$ & 40,6 & 44,9 \\
\hline Ácido fólico ( $\mu \mathrm{g})$ & 670,8 & $449,9-919,5$ & 191,6 & 19,7 & 719,5 & $445,8-1064,3$ & 205,6 & 18,7 \\
\hline \multicolumn{9}{|l|}{ Nutrientes críticos por exceso } \\
\hline Azúcares simples (g) & 92,3 & $67,4-130,2$ & 174,2 & 23,4 & 107,5 & $(79,8-135,1)$ & 202,7 & 13,8 \\
\hline Sodio (mg) & 1344,4 & $846,5-2049,0$ & 67,2 & 9,1 & 1423,2 & $891,2-2110,5$ & 71,2 & 9,8 \\
\hline Grasas saturadas (g) & 21,5 & $15,3-30,8$ & 92,4 & 13,2 & 21,9 & $15,1-30,9$ & 93,8 & 12,9 \\
\hline Energía total (kcal) & 2233,9 & $1762,8-3080,4$ & 107,4 & 14,4 & 2168,8 & $1715,5-2680,4$ & 104,3 & 11,9 \\
\hline
\end{tabular}

Fuente: Elaboración propia.

IC95\%= Intervalo de confianza del 95\%; p25-p75= Percentiles 25 y 75 .

*Para la definición de la cobertura de la meta: se tomó como parámetro el valor de la mediana en todos los análisis. Las metas nutricionales se establecieron según los lineamientos de organismos internacionales. **Para la cobertura desde el comedor escolar (\%): se tomó como parámetro el valor de la mediana en todos los análisis. Se consideró el aporte de comedores escolares obtenido por el relevamiento y se estimó el porcentaje que este aporte representa en la ingesta total. 
Tabla 4. Mediana y percentiles 25 y 75 de nutrientes críticos de la ingesta nutricional de niñas y niños asistentes y no asistentes a comedores escolares. Escuelas municipales de Córdoba, Argentina, 2018.

\begin{tabular}{|c|c|c|c|c|c|c|}
\hline \multirow[t]{2}{*}{ Ingesta nutricional } & \multicolumn{3}{|c|}{$\begin{array}{l}\text { Asiste al comedor escolar } \\
\qquad(\mathrm{N}=140)\end{array}$} & \multicolumn{3}{|c|}{$\begin{array}{l}\text { No asiste al comedor escolar } \\
\qquad(\mathrm{N}=51)\end{array}$} \\
\hline & Mediana & p25-p75 & $\begin{array}{l}\text { Cobertura (\%) } \\
\text { de la meta* }\end{array}$ & Mediana & p25-p75 & $\begin{array}{l}\text { Cobertura (\% } \\
\text { de la meta* }\end{array}$ \\
\hline \multicolumn{7}{|c|}{ Nutrientes críticos por déficit } \\
\hline Vitamina A $(\mu \mathrm{g})$ & 308,1 & $209,0-452,0$ & 56,0 & 308,6 & $178,3-547,6$ & 56,1 \\
\hline Calcio (mg) & 438,8 & $323,5-629,4$ & 43,9 & 512,4 & $380,6-670,8$ & 51,2 \\
\hline Zinc (mg) & 11,9 & $8,5-15,1$ & 170,7 & 11,0 & $8,2-14,0$ & 156,7 \\
\hline Vitamina C (mg) & 32,2 & $14,0-64,4$ & 85,9 & 37,0 & $18,2-63,5$ & 98,6 \\
\hline Fibra (g) & 9,2 & $6,7-12,0$ & 32,1 & 9,2 & $6,5-12,6$ & 32,3 \\
\hline Hierro (mg) & 16,2 & $12,0-22,1$ & 130,0 & 15,4 & $11,9-22,1$ & 123,4 \\
\hline Vitamina D $(\mu \mathrm{g})$ & 2,0 & $1,0-3,1$ & 40,2 & 2,2 & $1,0-3,4$ & 43,8 \\
\hline Ácido fólico ( $\mu \mathrm{g})$ & 725,3 & $452,5-1099,0$ & 207,2 & 671,5 & $393,9-940,6$ & 191,9 \\
\hline \multicolumn{7}{|c|}{ Nutrientes críticos por exceso } \\
\hline Azúcares simples (g) & 102,3 & $78,7-129,8$ & 192,9 & 115,7 & $87,1-139,1$ & 218,3 \\
\hline Sodio (mg) & 1446,3 & $895,4-2048,6$ & 72,3 & 1370,7 & $891,2-2308,5$ & 68,5 \\
\hline Grasas saturadas (g) & 21,5 & $14,8-29,5$ & 92,3 & 22,6 & $17,4-34,2$ & 96,8 \\
\hline Energía total (kcal) & 2154,5 & $1708,3-2639,1$ & 103,6 & 2302,2 & $1757,3-2777,4$ & 110,7 \\
\hline
\end{tabular}

Fuente: Elaboración propia.

* Para la definición de la cobertura de la meta: se tomó como parámetro el valor de la mediana en todos los análisis. Las metas nutricionales se establecieron según los lineamientos de organismos internacionales.

IC $95 \%=$ intervalo de confianza del $95 \%$; $\mathrm{p} 25-\mathrm{p} 75=$ percentiles 25 y 75 .

desde el comedor escolar este valor estaría cubriendo un $23 \%$ o menos de la mediana de ingesta global (dependiendo el nutriente), con excepción en la vitamina $\mathrm{D}$ que alcanzó un 48,3\% de cobertura (Tabla 3 ).

A partir del análisis de la cobertura de las metas nutricionales saludables recomendadas por organismos internacionales, se observó que una parte importante de los nutrientes bajo análisis en ambos periodos de análisis no alcanzaron la meta propuesta (tomando el valor de la mediana) siendo esto más marcado en el 2018. Los casos más críticos del insuficiente alcance de meta se observaron en torno al calcio, la vitamina A, fibra y vitamina $\mathrm{D}$, como así también de azúcares simples (Tabla 3).

Al realizar el análisis de subgrupos comparando la ingesta nutricional de niñas y niños asistentes y no asistentes al comedores escolares (Tabla 4) durante el relevamiento 2018, se pudo observar que una parte de los indicadores nutricionales fueron mas bajos en el grupo bajo cobertura de comedores escolares con respecto a los que no asistieron, en especial en calcio donde la mediana en asistentes fue de 438,8 y en no asistentes de 512,4 , mientras que en la vitamina $C$ se encontró una mediana de 32,2 y 37,0 y vitamina D una mediana 2,0 y 2,2 , entre asistentes/no asistentes respectivamente. En los nutrientes críticos por exceso, se observó una ingesta según la mediana mas alta de sodio total en el subgrupo asistente que no asistente. Sin embargo, se observó que la energía total fue menor en el grupo asistente versus el no asistente (Tabla 4).

Sobre el alcance de las metas nutricionales saludables, se observó que una parte importante de los nutrientes bajo análisis, en especial el calcio, la vitamina $C$ y la vitamina $\mathrm{D}$, tanto en el grupo asistente como no asistente fue inadecuado, aunque se observó que este escenario fue más notorio en el subgrupo que asistió al comedor escolar.

En la Tabla 5, se incorpora una columna que representa la proporción de niñas y niños con "diagnóstico adecuado" tanto en el subgrupo de asistentes como no asistentes al comedor escolar en cada uno de los Z-score bajo análisis (IMC/edad, talla/edad y peso/ edad) siguiendo los lineamientos de la OMS. En cuanto al estado nutricional, se observaron diferencias en los percentiles en el subgrupo asistente versus no asistente al comedor escolar como, por ejemplo, en el caso del Z-score 
Tabla 5. Z-score del estado nutricional de niñas y niños asistentes y no asistentes a comedores escolares. Escuelas municipales de Córdoba, Argentina, 2018.

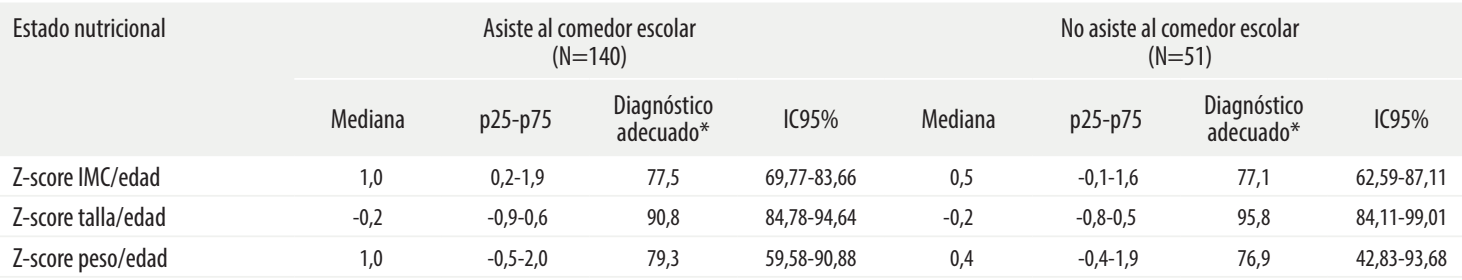

Fuente: Elaboración propia.

*El criterio tomado para establecer "diagnóstico adecuado" en el Z-score IMC/edad fue la categoría peso adecuado, en Z-score talla/edad fue talla adecuada, y en Z-score peso/edad fue el peso adecuado.

IC95\%= intervalo de confianza del 95\%; p25-p75= percentiles 25 y 75 .

peso/edad. El primer grupo tuvo una mediana de $1,0 \mathrm{y}$, en el segundo, una mediana de 0,4 . El percentil talla/edad en el grupo asistente tuvo una proporción de la muestra con diagnóstico adecuado del $90,8 \%$ y un $95,8 \%$ en el no asistente, mientras que el percentil IMC/ edad fue más elevado en el primer grupo con respecto al segundo (Tabla 5).
Como muestra la Tabla 6, en los modelos de regresión logística simple y múltiples ajustados por potenciales confusores sobre la relación entre la categoría de no alcanzar las metas nutricionales saludables y la asistencia al comedor, se encontraron OR de $1,37$ [IC95\% $(0,47-3,98) ; p=0,566]$ en grasas saturadas y OR de 1,42 [IC95\% $(0,62-3,28)$;

Tabla 6. Odds ratio entre el alcance de metas nutricionales y el estado nutricional, según Z-score de niñas y niños y la asistencia a comedores escolares. Escuelas municipales de Córdoba, Argentina, 2018. $(n=191)$

\begin{tabular}{|c|c|c|c|c|c|c|}
\hline \multirow[t]{2}{*}{ Ingesta nutricional $(n=190)$} & \multicolumn{3}{|c|}{ Modelo 1} & \multicolumn{3}{|c|}{ Modelo 2} \\
\hline & $O R^{*}$ & IC95\% & Valor de $p$ & $O R^{*}$ & IC95\% & Valor de $p$ \\
\hline \multicolumn{7}{|l|}{ Nutrientes críticos por déficit } \\
\hline Vitamina A $(\mu \mathrm{g})$ & 1,19 & $0,33-4,29$ & 0,789 & 0,80 & $0,12-5,12$ & 0,812 \\
\hline Calcio (mg) & 1,00 & - & - & 1,00 & - & - \\
\hline Zinc (mg) & 0,90 & $0,30-2,67$ & 0,846 & 0,53 & $0,10-2,92$ & 0,470 \\
\hline Vitamina C (mg) & 1,15 & $0,59-2,24$ & 0,674 & 1,23 & $0,48-3,12$ & 0,669 \\
\hline Fibra (g) & 3,50 & $0,20-60,84$ & 0,390 & 1,00 & - & - \\
\hline Hierro (mg) & 0,80 & $0,36-1,74$ & 0,570 & 0,53 & $0,24-1,16$ & 0,112 \\
\hline Vitamina D $(\mu \mathrm{g})$ & 0,21 & $0,03-1,56$ & 0,128 & 1,00 & - & - \\
\hline Ácido fólico $(\mu \mathrm{g})$ & 1,19 & $0,54-2,59$ & 0,663 & 1,27 & $0,48-3,37$ & 0,627 \\
\hline \multicolumn{7}{|l|}{ Nutrientes críticos por exceso } \\
\hline Azúcares simples (g) & 0,58 & $0,23-1,50$ & 0,263 & 0,80 & $0,29-2,23$ & 0,674 \\
\hline Sodio (mg) & 0,75 & $0,48-1,17$ & 0,208 & 0,65 & $0,33-1,27$ & 0,207 \\
\hline Grasas saturadas (g) & 0,90 & $0,56-1,47$ & 0,684 & 1,37 & $0,47-3,98$ & 0,566 \\
\hline Energía total (kcal) & 1,05 & $0,49-2,21$ & 0,906 & 1,42 & $0,62-3,28$ & 0,406 \\
\hline Estado nutricional (Z-score) & $O \mathrm{R}^{* *}$ & $\mathrm{IC95 \%}$ & Valor de $p$ & $O R^{* *}$ & IC95\% & Valor de $p$ \\
\hline Z-score IMC/edad ( $n=190)$ & 0,98 & $0,50-1,91$ & 0,949 & 0,94 & $0,45-1,96$ & 0,869 \\
\hline Z-score talla/edad $(n=190)$ & 2,32 & $0,49-10,99$ & 0,290 & 2,59 & $0,53-12,80$ & 0,241 \\
\hline Z-score peso/edad $(n=42)$ & 0,87 & $0,14-5,47$ & 0,882 & 1,24 & $0,18-8,54$ & 0,829 \\
\hline
\end{tabular}

${ }^{*} \mathrm{OR}=$ odds ratio obtenido por un modelo de regresión logística, donde las variables dependientes fueron el alcance de meta nutricional (categorías: alcanza/no alcanza) de todos los nutrientes bajo análisis por déficit y exceso. La variable independiente fue la asistencia al comedor escolar. Valor de $p=$ nivel de significancia estadística $<0,05$. IC95\%= intervalo de confianza del 95\%. Modelo 1: modelo bivariado crudo. Modelo 2: modelo 1 + ajuste por sexo (femenino/masculino), edad (dicotómica: hasta 10 años/más de 10 años), turno de asistencia escolar (mañana/tarde) y energía total (excepto en el modelo de alcance de meta de energía).

${ }^{* *} \mathrm{OR}=$ odds ratio obtenido por un modelo de regresión logística, donde las variables dependientes fueron el Z-score IMC/edad (categorías: peso adecuado/peso alto o más) Z-score talla/edad (categorías: talla adecuada 0 más/baja talla y riesgo de baja talla) y Z-score peso/edad (categorías: peso adecuado/peso alto o más). La variable independiente fue la asistencia al comedor escolar. Valor de $p=$ nivel de significancia estadística <0,05. IC95\%: intervalo de confianza del 95\%. Modelo 1: modelo bivariado crudo. Modelo 2: modelo $1+$ ajuste por número de miembros de la familia del niños o de la niña (variable continua) y turno de asistencia escolar (mañana/tarde). 
$p=0,406]$ en la energía total, aunque estas asociaciones no fueron estadísticamente significativas. También se vieron OR aumentados, aunque no significativas, en el no alcance de las metas de vitamina $\mathrm{C}$ y ácido fólico en los asistentes a comedores escolares.

Cuando se analizaron las odds ratio entre indicadores del estado nutricional de niñas y niños y la asistencia al comedor escolar, tanto en modelos crudos como ajustados, no se observaron diferencias estadísticamente significativas en ningún escenario. Aunque se encontró un OR de 2,59 [IC95\% (0,53-12,80)] en la categoría de baja talla/riesgo de baja talla en niñas y niños asistentes a comedores escolares en el modelo ajustado, no siendo estadísticamente significativo $(p=0,241)$ (Tabla 6).

\section{DISCUSIÓN}

A partir de los resultados alcanzados se concluye que sería de utilidad impulsar una política de comedores escolares que busque de manera urgente mejoras en los indicadores de calidad de los servicios alimentarios, como así también en los indicadores de intervención en la alimentación y nutrición infantil. Si bien los comedores escolares aportan una parte de los nutrientes críticos (tanto por déficit como por exceso) de la ingesta media global de niños y niñas asistentes a comedores escolares, en este grupo se encontraron indicadores más agravados de exceso de peso y baja taIla respecto de los no asistentes a comedores escolares, con un deterioro en los aportes nutricionales del comedor escolar, como así también en las ingestas entre el 2013 y 2018.

El déficit de micronutrientes generalizado $y$, por consiguiente, una baja presencia de alimentos fuentes como frutas y verduras, se vio plasmado de manera alarmante tanto en este trabajo como en otro estudio previo(38).

El contexto de desigualdades en el que se inserta el comedor escolar, aparentemente, estaría condicionando los aportes nutricionales. Incorporar dentro del análisis este enfoque es relevante al momento de pensar la nutrición infantil desde un enfoque integral y en el marco de derechos. En este sentido, se observó que en el subgrupo de comedores escolares insertos en entornos con nivel de hacinamiento crítico -tanto en el relevamiento llevado a cabo en 2013 como en 2018- la mayoría de los nutrientes críticos y energía total aportados fueron menores. Este hallazgo es un Ilamado de atención acerca de la importancia de proteger el derecho a la alimentación saludable desde las políticas públicas en todos los grupos sociales, en especial, los grupos con mayor vulnerabilidad social y más aún en un escenario de crisis y aumento de los indicadores de pobreza, dejando una puerta abierta para futuros estudios que consideren variables de desigualdades, más aún considerando que los grupos más vulnerables son los destinatarios directos de estas políticas. En consonancia con nuestros hallazgos y según datos de 2019 del Informe del Barómetro de Deuda Social de la Infancia de la Universidad Católica Argentina, la pobreza extrema por indigencia monetaria y/o privaciones graves afectó, en 2018, al 24,7\% de la infancia entre 0 y 17 años ${ }^{(39)}$.

Las diferencias antes mencionadas entre el empobrecimiento de la política de comedores escolares entre 2013 y 2018 puede estar relacionada al contexto económico y social del país, con el aumento de los indicadores de pobreza de $2018^{(39)}$. Si bien el menú de los comedores escolares, relevados durante ambos periodos, aparentemente, se mantuvo sin modificaciones en el periodo bajo análisis, la reducción de los aportes globales de los nutrientes analizados pudo deberse a una reducción del tamaño de las porciones, al reemplazo de ingredientes, o bien a un aumento de los destinatarios que asistieron al momento del servido, impactando en el aporte global de nutrientes por parte de la política. Entender la intervención pública desde el territorio y la realidad local en un contexto nacional y el potencial rol dentro de los procesos alimentarios nutricionales de niñas y niños en el contexto de privaciones y desde un enfoque multidimensional será crucial en el alcance pleno del derecho a la alimentación. 
La malnutrición infantil en usuarios frecuentes de programas alimentarios escolares debería ser prioritaria en la agenda pública de los países de América Latina y el Caribe y a nivel del país en un contexto actual de indicadores alarmantes de malnutrición ${ }^{(40)}$.

Los hallazgos de esta investigación estarían indicando que los alimentos más consumidos por escolares pertenecieron, en más de un tercio, al grupo de opcionales (según las GAPA) ${ }^{(30)}$, siendo los que más aportan nutrientes críticos por excesos, necesarios de reducir en el marco de las políticas de nutrición saludable. Estos resultados son similares a lo reportado por la ENNyS, 2018-2019(2), y parecidos a los publicados recientemente por Dunford y Popkin ${ }^{(41)}$ en niñas y niños de 2 a 18 años en EEUU. Al analizar específicamente la ingesta de nutrientes en el trabajo de Dunford y Popkin, se observaron múltiples inadecuaciones nutricionales en la ingesta de niñas y niños, siendo el calcio, la fibra, la vitamina A y los azúcares simples los más alarmantes en ambos periodos de relevamiento, inadecuaciones que también se reportaron de manera similar en los datos nacionales ${ }^{(2)}$.

Se visualizó un empeoramiento de los indicadores en una parte importante de los nutrientes bajo análisis entre 2013 y 2018. Sin embargo, las diferencias estadísticamente significativas se encontraron en la ingesta media de calcio y energía total, donde fueron superiores en el primer relevamiento en comparación con el segundo. Como se hizo referencia en el apartado de análisis de la calidad nutricional de los servicios alimentarios de comedores escolares, estos resultados pueden deberse al contexto de un aumento de los indicadores de pobreza infantil( ${ }^{(39)}$ que afecta de manera directa el acceso a una nutrición saludable y suficiente.

Vincular las situaciones de privaciones y el contexto social resulta fundamental para entender los resultados nutricionales, como lo demostró el estudio de Monteiro et al. en Brasil ${ }^{(42)}$ durante el periodo 1974-2007, el desarrollo socioeconómico junto con políticas públicas orientadas a la equidad han sido acompañados por mejoras notables en las condiciones de vida y una disminución sustancial en la desnutrición infantil, así como una reducción de la brecha en el estado nutricional de los niños en los quintiles socioeconómicos más altos y más bajos.

En esta misma línea, un estudio que analizó la malnutrición y las disparidades mostró cómo el exceso de peso es más prevalente en países de bajos y medianos ingresos, donde dentro de los más afectados se encuentran las niñas y los niños ${ }^{(43)}$ y también se pone el peso de las desigualdades sociales y económicas en el retraso del crecimiento a lo largo del tiempo ${ }^{(44)}$. Además, según un estudio longitudinal realizado en 2009, el retraso en el crecimiento en niños y niñas se asoció con una menor escolarización, un menor rendimiento escolar y un aumento de la probabilidad de vivir en la pobreza durante la vida adulta ${ }^{(45)}$. Esto indica cómo la malnutrición infantil puede ser causa y efecto de situaciones de vulnerabilidad, donde las políticas públicas son cruciales para intervenir en estos escenarios complejos.

Como se encontró en este trabajo una parte importante de las niñas y los niños, tanto en el grupo de asistentes como no asistentes a comedores escolares no alcanzaron las metas nutricionales saludables para su edad. Aunque dentro del subgrupo que asistió al comedor se encontró que las ingestas medias de algunos nutrientes críticos como la vitamina $A$ y el calcio y el total de energía fueron menores que en el grupo que no asistió, como así también un deterioro de los indicadores del estado nutricional, en especial, del exceso de peso y de baja talla/riesgo de baja talla, aunque en líneas generales, en ambos grupos los perfiles nutricionales fueron poco óptimos. Este hallazgo permite dar cuenta y verificar que en los comedores y en los hogares se come lo mismo, de manera que la política que intenta ser "compensadora" se transforma en "conservadora", en términos de cantidad y no calidad, como ya lo plantearon de manera similar diferentes autores $^{(15,17,27)}$, Ilevando a plantear la necesidad apremiante de mejorar la política de alimentación en los entornos escolares.

En cuanto a la relación explorada entre la ingesta de las niñas y los niños y la asistencia 
al comedor escolar (modelos ajustados por potenciales confusores) si bien no se encontraron asociaciones estadísticamente significativas, se observó que en nutrientes críticos por exceso, en el grupo expuesto al comedor escolar, las grasas saturadas tuvieron un OR de 1,37 y la energía total un OR de 1,42, y también se vio un OR aumentado en el no alcance de las metas de otros nutrientes críticos por déficit, como la vitamina $\mathrm{C}$ y el ácido fólico en los asistentes a comedores escolares.

Este resultado es relevante ya que deja la puerta abierta para profundizar, desde estudios longitudinales, sobre el peso que tiene la política de comedores escolares en el exceso de nutrientes críticos en la ingesta. Como lo postula la OMS, estos nutrientes se han asociado con diversas enfermedades no transmisibles ${ }^{(46)}$, marcando mayores riesgos para el desarrollo futuro de enfermedades crónicas en niñas y niños que hayan tenido parte de su alimentación cubierta por los comedores escolares. Son pocos los antecedentes publicados en esta línea. Un estudio previó indicó que la ingesta de energía, carbohidratos y grasas en el grupo que asistió al comedor escolar fue significativamente mayor que en los que no asistieron ${ }^{(19)}$ aunque se basó en un análisis bivariado.

Un estudio realizado por Ongan et al. no encontró diferencias estadísticamente significativas en peso corporal, altura y el índice de masa corporal en el grupo que asistió al comedores escolares con respecto a los que no asistieron ${ }^{(22)}$ aunque en este estudio no se reportan variables de confusión.

En Argentina, encontramos algunos estudios aislados que analizan aspectos de programas de comedores escolares en escuelas públicas y describen las características de los servicios $^{(12,13,14,28)}$, aunque son limitados los análisis más robustos que den cuenta de la intervención sobre la nutrición infantil. Lamentablemente, esta limitación también se hace extensiva a varios de los países de América Latina ${ }^{(47)}$.

Una excepción a este panorama podría ser el estudio publicado por Rausch Herscovici et al. ${ }^{(48)}$, que, dentro de varias intervenciones, se centró en proveer alimentos de mejor valor nutricional para ser ofrecidos en los comedores escolares, en el marco de otras intervenciones de educación y actividad física. Si bien los autores encontraron cambios significativos en algunos indicadores de consumo de alimentos saludables tuvo la limitación que la intervención fue de corto periodo (seis meses), como lo plantearon los propios autores $^{(48)}$, y no se exploraron aspectos sobre la exposición al comedor escolar en variables de resultado, como es el alcance de metas nutricionales de niños y niñas.

Otro resultado que será necesario profundizar en futuros estudios es la asociación entre estar expuesto a comedores escolares y los indicadores de estado nutricional. En esta línea encontramos un OR de 2,59 en la categoría de baja talla/riesgo de baja talla en el subgrupo que estuvo expuesto a comedores escolares respecto al que no, aunque esto no fue estadísticamente significativo. El no encontrar diferencias significativas en el análisis multivariado pudo deberse a que el tamaño muestral del subgrupo que no asistió a comedores escolares fue pequeño con intervalos de confianza grandes. Además, como lo plantea Galván et al. ${ }^{(49)}$ los impactos en indicadores de desnutrición crónica necesitan ser analizados a largo plazo.

En el marco de un avance de la región en promover esfuerzos en intervenciones en entornos escolares y que estas sean evaluadas, diferentes organismos internacionales vienen instalando los entornos escolares saludables en la agenda global, regional y nacional, como punto de partida en la lucha contra el sobrepeso, obesidad y malnutrición infanti ${ }^{(50)}$. Sin embargo, no se disponen de datos aparentes y actualizados sobre las acciones a nivel de las provincias y municipios de Argentina con relación a este tema y existe una ausencia de evaluaciones de impacto que puedan dar cuenta de los esfuerzos llevados a cabo. Esto ya fue puesto en relieve en un estudio previo ${ }^{(27)}$.

La bibliografía a nivel mundial, estaría indicando el potencial que tienen los programas de comedores escolares en mejorar los indicadores nutricionales en la población 
infanto-juvenil. Una revisión publicada en el 2016 por Meiklejohn et al., que evaluó el impacto de las intervenciones nutricionales en adolescentes, evidenció que los cambios en las cantinas escolares se asociaron con cambios significativos en la ingesta alimentaria ${ }^{(51)}$. También se destacan aportes a la permanencia en la escuela, el rendimiento escolar, implicancias sobre la protección social y la seguridad alimentaria y nutricional individual y familiar, efectos positivos en torno a los hábitos saludables y en los procesos de socialización de niñas y niños ${ }^{(52,53)}$.

Sin embargo, en la región existen escasos antecedentes publicados sobre el aporte real que tienen los comedores escolares y más aún desde intervenciones saludables. En los últimos años a nivel de América Latina existen otras propuestas alternativas para comedores escolares ${ }^{(52,53)}$. Uno de los casos más sobresalientes es el de Brasil que, a pesar de tener un programa alimentario escolar de larga data, se están Ilevando adelante en la actualidad acciones que trascienden el carácter asistencialista que históricamente han tenido estos programas ${ }^{(54)}$.

Hoy cuenta con su respectivo marco legal y busca contribuir de manera favorable al crecimiento, desarrollo biopsicosocial, estado nutricional, aprendizaje y rendimiento escolar de los niños, como así también promover hábitos alimentarios saludables, actuar sobre factores ambientales adversos, incorporar instancias de educación alimentaria y nutricional (EAN) y fortalecer la participación de la comunidad local en el control y gestión del programa ${ }^{(54)}$.

Entre las limitaciones de este trabajo pueden señalarse los sesgos provenientes de estudios transversales, como el selección que podría haber existido al momento de excluir a los escolares con algún deterioro cognitivo y/o intelectual, aunque datos oficiales ${ }^{(55)}$ estarían indicando que el porcentaje de niñas y niños con discapacidad que están incluidos hoy en escuelas comunes es bajo. También se pudo dar la presencia de sesgo de información y de complacencia social (información) frecuente en estudios poblacionales que relevan datos alimentarios; sin embargo, los datos se recolectaron a través de un cuestionario con preguntas abiertas, guiado por investigadores entrenados, lo que podría disminuir la influencia de este sesgo.

En las relaciones exploradas, pudo existir el sesgo de confusión y de causalidad reversa común en estudios trasversales epidemiológicos, aunque contamos con un grupo de comparación que permitió realizar comparaciones y se realizaron modelos ajustados por potenciales confusores. Además, la muestra pequeña pudo ser una limitación al momento de analizar las relaciones, por lo que se necesitará realizar futuros estudios más robustos, con muestras más grandes que permitan mejorar las estimaciones. Sin embargo, contar con datos en dos periodos temporales (2013 y 2018), en diferentes contextos sociopolíticos del país e incluir un análisis comparativo y desde un enfoque de desigualdades, es clave al momento de definir la intervención pública en pos del alcance del derecho a la alimentación y otros derechos fundamentales, como de la salud y la educación.

Este estudio tiene múltiples e importantes fortalezas en el campo de la salud pública y las políticas públicas. La muestra representativa de las escuelas municipales bajo análisis con un suficiente número de niñas y niños que permitió hacer estimaciones poblacionales. Además, esta propuesta aporta evidencia a un tema hoy controvertido y con múltiples vacíos de conocimiento en nuestra región.

A continuación, se detallan algunas recomendaciones relacionadas con el tema abordado desde este estudio y con futuras líneas de trabajo en el contexto nacional y local.

\section{A nivel de la gestión e implementación de la política de comedores escolares}

- Evaluar el diseño e implementación del programa, considerando la variable de un estándar mínimo de calidad nutricional de los servicios alimentarios brindados, ya que se observaron múltiples inadecuaciones nutricionales.

- Realizar nuevos relevamientos que permitan mejorar los registros y analizar de manera longitudinal indicadores de monitoreo. 
- Realizar capacitación continua al personal del comedor, basada en un enfoque de procesos de mejora, principalmente en la elaboración de desayunos y meriendas, donde se detectó de manera crítica el déficit de micronutrientes, en especial de calcio y el análisis de la oferta de raciones que sea suficiente y de calidad para toda la población que asiste diariamente.

- Revisar la posibilidad de incluir dentro del programa otros aspectos relacionados con la salud escolar y la articulación entre programas públicos, principalmente, para el seguimiento de niños y niñas en riesgo nutricional.

- Brindar talleres de análisis y educación sobre alimentación saludable entre diferentes actores, en el que se incluyan a niñas y niños, docentes y las familias, ya que se constató que la alimentación escolar como extra-escolar necesitan mejorarse con urgencia.

- Evaluar la posibilidad de aumentar el aporte de frutas y verduras, siendo estos alimentos los más deficitarios en el menú de los comedores escolares según este relevamiento, y promover principalmente que estos alimentos provengan desde productores locales. Como lo reportó FAO en un informe, el uso de compras locales para comedores escolares proviene de la agricultura familiar y de pequeños agricultores, en países como Guatemala, Jamaica, Perú, Ecuador, Nicaragua, Uruguay, Brasil, Barbados, Colombia, Guyana, Bolivia, Paraguay, Granada, Belice, Trinidad y Tobago ${ }^{(56)}$, por lo que ya existirían experiencias previas sobre este aspecto y sería factible de ser implementadas.

\section{A nivel del impacto de la política de comedores escolares}

- Desarrollar futuros estudios de impacto desde enfoques cuanti-cualitativos de la política de comedores escolares en diferentes grupos y en mayor profundidad sobre las relaciones exploradas, como así también sus mecanismos vinculantes y sus beneficios psicosociales y sanitarios, a partir de diseños más robustos.

- Considerar la posibilidad de relevar datos sobre la asistencia a comedores escolares y característica de los destinatarios en el marco de las encuestas nacionales de salud, por ejemplo: la Encuesta Mundial de Salud Escolar y la Encuesta Nacional de Nutrición y Salud que se implementan en nuestro país.

\section{A nivel de las regulaciones, políticas públicas locales, subnacional y nacional de comedores escolares}

- Iniciar un proceso de legislación y marcos regulatorios a nivel local específicos de comedores escolares, que considere una revisión periódica de los estándares de calidad de los servicios y de los alcances multidimensionales de la política.

- Evaluar la posibilidad de elaborar una guía marco de referencia nacional de buenas prácticas nutricionales en comedores escolares como es el caso de la experiencia de Granada y Brasil que ya tienen una guía de referencia, y donde las personas que preparan los alimentos son capacitadas en su uso para garantizar que los requerimientos se cumplan ${ }^{(56)}$.

- Este trabajo no tuvo dentro de sus objetivos el análisis de los marcos jurídicos-normativos actuales que garantizan el derecho a la alimentación, aunque se recomienda profundizar sobre la Ley 25724 que crea el Programa de Nutrición y Alimentación Nacional y rever -entre varios aspectossu nivel de cumplimiento en la línea de comedores escolares; después de casi 18 años de su sanción, reflexionar sobre potenciales redefiniciones adecuadas al contexto actual y analizar la implementación del Artículo 4, que crea la Comisión Nacional de Nutrición y Alimentación y la reglamentación de los Artículos 7 y 8, que abordan la creación de comisiones de los gobiernos provinciales y las funciones de los municipios, siendo aspectos fundamentales en el marco de un país federal. 


\section{AGRADECIMIENTOS}

A la Universidad Nacional de la Matanza a través del Programa "Formando UNLaM", 2019 por los fondos aportados para la etapa de análisis de este trabajo y a la Dirección de Investigación para la Salud, Becas Salud Investiga, 2013 y 2018 por los fondos para la realización de las dos etapas de campo. Al Dr. Jorge Chaui y a la Dra. Paula Vázquez por la revisión de los análisis.

\section{REFERENCIAS BIBLIOGRÁFICAS}

1. Organización de las Naciones Unidas para la Alientación y la Agricultura, Organización Panamericana de la Salud, Programa Mundial de Alimentos, UNICEF. Panorama de la seguridad alimentaria y nutricional en América Latina y el Caribe. Santiago: FAO, OPS, WFP y UNICEF; 2018.

2. Secretaría de Gobierno de Salud, Ministerio de Salud y Desarrollo Social. $2^{\circ}$ Encuesta Nacional de Nutrición y Salud (ENNyS 2). Buenos Aires: Ministerio de Salud y Desarrollo Social; 2019.

3. Kac G, García Alvear JL. Epidemiología de la desnutrición en Latinoamérica: situación actual. Nutrición Hospitalaria. 2010;25(Supl 3):S50-S56.

4. Ministerio de Salud de la Nación. Encuesta Mundial de Salud Escolar 2018 [Internet]. Buenos Aires: Ministerio de Salud y Desarrollo Social; 2018 [citado 10 mar 2019]. Disponible en: Disponible https://tinyurl.com/ y4gp2mle.

5. Veleda C, Repetto F, Díaz Langou G, Bezem P, Sánchez B, Cano E. Comer en la escuela: nueve acciones para mejorar la gestión de los comedores escolares [Internet]. CIPPEC; 2014 [citado 10 mar 2019]. Disponible en: https://tinyurl.com/y287njol.

6. Cohen E, Franco R. Seguimiento y evaluación de impacto de los programas de protección social basados en alimentos en América latina y el Caribe [Internet]. Santiago; 2005 [citado 10 mar 2019]. Disponible en: https:// tinyurl.com/y4897q7b.

7. Reilly K, Nathan N, Grady A, Wu J, Wiggers J, Yoong $\mathrm{SL}$, Wolfenden L. Barriers to implementation of a healthy canteen policy: A survey using the theoretical domains framework. Health Promotion Journal of Australia. 2019;(Suppl 1):S9-S14. doi: 10.1002/hpja.218.

8. Varman S, Bullen C, Tayler-Smith K, Van Den Bergh R, Khogali M. Primary school compliance with school canteen guidelines in Fiji and its association with student obesity. Public Health Action. 2013;3(1):81-84. doi: 10.5588/pha.12.0063.

9. Gabriel CG, Vasconcelos FA, Andrade DF, Schmitz BA. First law regulating school canteens in Brazil: evaluation after seven years of implementation. Archivos Latinoamericanos de Nutrición. 2009;59(2):128-138.

10. Drummond C, Sheppard L. Examining primary and secondary school canteens and their place within the school system: a South Australian study. Health Edu- cation Research. 2011;26(4):739-749. doi: 10.1093/her/ cyr036.

11. Ministerio de Sanidad y Política Social. Programa piloto escolar de referencia para la salud y el ejercicio contra la obesidad - Programa PERSEO [Internet]. s/f. [citado 10 mar 2019]. Disponible en: https://tinyurl.com/ yxzhsyx8.

12. Moyano D, Perovic NR. Nutritional contribution the school canteens program to the children of ten municipal schools in the city of Cordoba, Argentina. Revista de la Facultad de Ciencias Medicas. 2018;75(3):194-202.

13. Buamden S, Graciano A, Manzano G, Zummer E. Proyecto "Encuesta a los Servicios Alimentarios de Comedores Escolares Estatales" (PESCE): alcance de las metas nutricionales de las prestaciones alimentarias de los comedores escolares de Gran Buenos Aires, Argentina. Diaeta (Buenos Aires). 2010;28(130):21-30.

14. Bracamonte S, Carranza C, Olivero I, Laquis M. Calidad nutricional y consumo de los menúes del comedor escolar y estado nutricional de los alumnos del nivel primario de la escuela Jorge Peyrano, Ciudad de La Calera, Provincia de Córdoba, 2012. Córdoba: Escuela de Nutrición, Universidad Nacional de Córdoba; 2012.

15. Aguirre P. Pobres gordos, ricos flacos: la alimentación en crisis. Buenos Aires: Capital Intelectual; 2004.

16. Aguirre P. Reflexiones sobre las nuevas formas del hambre en el siglo XXI: La obesidad de la escasez. Boletín Científico Sapiens Research. 2011;1(2):60-64.

17. Del Castillo SE. La situación nutricional de la niñez en Latinoamérica: entre la deficiencia y el exceso, de brecha nutricional a deuda social. Biomédica. 2012;32(4):471473.

18. Clinton-McHarg $\mathrm{T}$, Janssen L, Delaney $\mathrm{T}$, Reilly $\mathrm{K}$, Regan T, Nathan N, et al. Availability of food and beverage items on school canteen menus and association with items purchased by children of primary-school age. Public Health Nutrition. 2018;21(15):2907-2914.

19. Ministerio de Sanidad y Política Social. Informes técnicos de evaluación del programa PERSEO de promoción de la alimentación y la actividad física saludables en el ámbito escolar. España: AESAN; 2009.

20. Reilly KL, Nathan N, Wiggers J, Yoong SL, Wolfenden L. Scale up of a multi-strategic intervention to increase implementation of a school healthy canteen policy: findings of an intervention trial. BMC Public Health. 2018;18(1):860.

21. Garbayo Solana J, Tomás Herrero N, Navarro Elizondo M, Sánchez Hernández B, Murillo Zardoya R, Lorente Simon $\mathrm{M}$, et al. C-22. Influencia de los comedores escolares en la nutrición de niños de 4 a 6 años. Pediatría Atención Primaria. 2011;13(Supl 20):S40-S41.

22. Ongan D, Inanc N, Cicek B. Comparing school lunch and canteen foods consumption of children in kayseri, Turkey. Pakistan Journal of Medical Sciences. 2014;30(3):549-553. 
23. Campos Díaz J, Rodríguez Álvarez C, Calvo Pacheco M, Arévalo Morales MP, Sierra López A, Arias Rodríguez A. Valoración nutricional de los menús escolares de los colegios públicos de la isla de Tenerife. Nutrición Hospitalaria. 2008;23:41-45.

24. Castro M, Ríos-Reina R, Ubeda C, Callejón RM. Evaluación de menús ofertados em comedores escolares: comparación entre colegios públicos, privados y concertados. Revista de Nutrição. 2016;29:97-108.

25. Centro de Implementación de Políticas Públicas para la Equidad y el Crecimiento (CIPPEC). Comer en la escuela: nueve acciones para mejorar la gestión de los comedores escolares. Buenos Aires: CIPPEC; 2014.

26. Organización Panamericana de la Salud. Evaluación de un programa de alimentación escolar: el caso argentino. Washington: OPS/OMS; 1990.

27. Abeyá Gilardon EO. Una evaluación crítica de los programas alimentarios en Argentina. Salud Colectiva. 2016;12(4):589-604.

28. Britos S, Virgolini M, Saraví A, Chichizola N, Moyano D, Perez L, Arroyo Parisi S. Análisis de la alimentación en el ámbito escolar. Anuario Becas de Investigación “Ramón Carrillo-Arturo Oñativia". 2013;(3):241.

29. Instituto Nacional de Estadística y Censos. Censo Nacional de Población, Hogares y Viviendas 2010 [Internet]. Buenos Aires: INDEC; 2010 [citado 10 may 2019]. Disponible en: https://tinyurl.com/y383xk6w.

30. Ministerio de Salud. Guías Alimentarias para la Población Argentina 2016 [Internet]. 2016 [citado 10 may 2019]. Disponible en: https://tinyurl.com/y5k9h3ku.

31. Ferrari M. Estimación de la ingesta por recordatorio de 24 horas. Diaeta (Buenos Aires). 2013;31(143):20-25.

32. Gibson RS. Principles of nutritional assessment. 2nd ed. New York: Oxford University Press; 2005.

33. Institute of Medicine FaNB. Dietary reference intakes (DRIs). Washington DC: National Academy Press; 2010.

34. World Health Organization, Food and Agriculture Organization. Expert consultation report on human energy requirements: Interin report. Rome: WHO, FAO; 2001.

35. World Health Organization, Food and Agriculture Organization. Consultation report on human vitamin and mineral requirements. Bangkok: WHO, FAO; 2001.

36. World Health Organization, Food and Agriculture Organization. Diet, nutrition and the prevention of chronic diseases. Geneva: WHO, FAO; 2003.

37. Sociedad Argentina de Pediatría, Comité Nacional de Crecimiento y Desarrollo. Guías para la evaluación del crecimiento físico 2013. 3a ed. Buenos Aires: SAP; 2013.

38. Diez González S, Rodríguez Bernardo C, Alonso Alonso O, Gutiérrez Diez MC, Allande Díaz R. Evaluación de la variedad y calidad en los menús escolares de Asturias. Curso 2015/2016. Revista Española de Salud Pública. 2018;92:e1-e12.

39. Tuñón I, Poy S. Pobreza, derechos e infancias en la Argentina (2010-2018). Ciudad Autónoma de Buenos Aires: Educa; 2019.

40. Organización Mundial de la Salud. Malnutrición [Internet]. 2017 [citado 10 mar 2019]. Disponible en: https://tinyurl.com/y6dxfrtn.

41. Dunford EK, Popkin BM. 37 year snacking trends for US children 1977-2014. Pediatric Obesity. 2018;13(4):247-255.

42. Monteiro CA, Benicio MH, Conde WL, Konno S, Lovadino $A L$, Barros $A J$, et al. Narrowing socioeconomic inequality in child stunting: the Brazilian experience, 1974-2007. Bulletin of the World Health Organization. 2010;88(4):305-311.

43. Perez-Escamilla R, Bermudez O, Buccini GS, Kumanyika $S$, Lutter $C K$, Monsivais $P$, et al. Nutrition disparities and the global burden of malnutrition. BMJ. 2018;361:k2252.

44. Restrepo-Mendez MC, Barros AJ, Black RE, Victora CG. Time trends in socio-economic inequalities in stunting prevalence: analyses of repeated national surveys. Public Health Nutrition. 2015;18(12):2097-2104.

45. Maluccio JA HJ, Behrman JR, Martorell R, Quisumbing AR, Stein AD. The impact of improving nutrition during early childhood on education among Guatemalan adults. The Economic Journal. 2009;119(537):734-763.

46. Organización Mundial de la Salud. Dieta, nutrición y prevención de enfermedades crónicas: informe de una consulta mixta de expertos OMS/FAO [Internet]. 2003 [citado 10 may 2019]. Disponible en https://tinyurl.com/ y2pukjfp.

47. Herscovic CR, Kovalskys I. Obesidad Infantil: Una revisión de las intervenciones preventivas en escuelas. Revista Mexicana de Trastornos Alimentarios. 2015;6(2):143-151.

48. Rausch Herscovici C, Kovalskys I, De Gregorio MJ. Gender differences and a school-based obesity prevention program in Argentina: a randomized trial. Revista Panamericana de Salud Pública. 2013;34(2):75-82.

49. Galván M, Amigo H. Programas destinados a disminuir la desnutrición crónica: Una revisión en América Latina. Archivos Latinoamericanos de Nutrición. 2007;57(4):316326.

50. Fondo de las Naciones Unidas para la Infancia. Situación de la Salud de las y los adolescentes de Argentina [Internet]. 2016 [citado 10 may 2019]. Disponible en: https://tinyurl.com/y38dxmqx.

51. Meiklejohn S, Ryan L, Palermo C. A systematic review of the impact of multi-strategy nutrition education programs on health and nutrition of adolescents. Journal of Nutrition Education and Behavior. 2016;48(9):631-646. 
52. Food and Agriculture Organization. School feeding and the possibilities for direct purchases from family farming - Case studies in eight countries. Santiago de Chile: FAO; 2013.

53. Food and Agriculture Organization, Brazilian Cooperation Agency. Strengthening school meal programmes under the Hunger Free Latin America and the Caribbean 2025 Initiative. Brazil: FAO, ABC; 2008.

54. Fundo Nacional de Desenvolvimiento da Educação. Programa Nacional de Alimentação Escolar (PNAE) [Internet]. 2012 [citado 10 may 2019]. Disponible en: https://tinyurl.com/yyymm2bd.
55. Ministerio de Educación. Información y Estadística educativa [Internet]. s/f [citado 10 mar 2019]. Disponible en: https://tinyurl.com/y3qjgaff.

56. Food and Agriculture Organization of the United Nations. Estudio para identificar experiencias nacionales relacionadas con el fortalecimiento de la educación alimentaria y nutricional en el marco de Programas de Nutrición y Alimentación Escolar [Internet]. 2018 [citado 10 may 2019]. Disponible en: https://tinyurl.com/y5av64e2.

\section{FORMA DE CITAR}

Moyano D, Rodríguez ER, Perovic NR. Análisis de la intervención de la política de comedores escolares y el rol en la nutrición saludable de niños y niñas de Córdoba, Argentina. Salud Colectiva. 2020;16:e2636. doi: 10.18294/sc.2020.2636.

Recibido: 16 nov 2019 | Versión final: 7 ago 2020 | Aprobado: 25 ago 2020 | Publicado en línea: 3 nov 2020

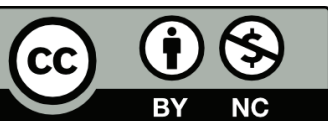

Esta obra está bajo una licencia de Creative Commons Reconocimiento-NoComercial 4.0 Internacional. Reconocimiento - Permite copiar, distribuir y comunicar públicamente la obra. A cambio, se debe reconocer y citar al autor original. No Comercial - Esta obra no puede ser utilizada con finalidades comerciales, a menos que se obtenga el permiso. 